\title{
Editorial Note: Semantic Approaches for Multimedia Retrieval Applications
}

Multimedia Tools and Applications gratefully acknowledges the editorial work of the scholars listed below on the special issue entitled, "Semantic Approaches for Multimedia Retrieval Applications."

Of 42 papers submitted to this issue, 11 were eventually accepted after a stringent peerreview process.

Dr. Chang Choi (Corresponding Guest Editor)

Chosun University

Gwangju, Rep. of Korea

enduranceaura@gmail.com

Prof. Marek R. Ogiela

AGH University of Science and Technology

Krakow, Poland

mogiela@agh.edu.pl

Dr. Christian Esposito

University of Salerno

Salerno, Italy

christian.esposito@dia.unisa.it 